\title{
Pour une critique des Médias en temps de Conflit?
}

(2)

For a critique of Media in time of War? (2)

Béatrice Fleury et Jacques Walter

\section{OpenEdition}

1 Journals

Édition électronique

URL : http://journals.openedition.org/questionsdecommunication/7698

DOI : 10.4000/questionsdecommunication.7698

ISSN : 2259-8901

Éditeur

Presses universitaires de Lorraine

Édition imprimée

Date de publication : 1 décembre 2006

Pagination : 181-195

ISBN : 978-2-86480-828-2

ISSN : 1633-5961

Référence électronique

Béatrice Fleury et Jacques Walter, "Pour une critique des Médias en temps de Conflit ? (2)»,

Questions de communication [En ligne], 10 | 2006, mis en ligne le 01 décembre 2006, consulté le 22

mars 2021. URL : http://journals.openedition.org/questionsdecommunication/7698; DOI : https:// doi.org/10.4000/questionsdecommunication.7698 


\title{
$>$ ÉCHANGES
}

\author{
BÉATRICE FLEURY \\ Université Nancy 2 \\ Centre de recherche sur les médiations \\ Université Paul Verlaine-Metz \\ Beatrice.Fleury@univ-nancy2.fr
}

JACQUES WALTER

Centre de recherche sur les médiations

Université Paul Verlaine-Metz

jacques.walter@univ-metz.fr

\section{POUR UNE CRITIQUE DES MÉDIAS EN TEMPS DE CONFLIT ? (2)}

\begin{abstract}
Résumé. - À partir des contributions de Patrick Champagne, Estrella Israel Garzón, Michael Palmer, Claire Gabrielle Talon, Jean-François Tétu, fournies en réponse à l'entretien avec Daniel Dayan, mené par Béatrice Fleury et Jacques Walter (Questions de communication, 8, 2005), cet article met en relief les facteurs et théories convoqués pour analyser le passage qui s'opère entre l'événement et sa mise en forme. Ce qui sera fait tant en examinant le lien entre discours politique et contraintes professionnelles qu'en s'attachant aux transformations des préoccupations sociales. Ceci est d'autant plus caractéristique que, dans ces «Échanges », s'expriment des chercheurs qui, tout en étant de traditions disciplinaires et/ou culturelles différentes (schématiquement, ils se rattachent à deux pôles: I'un proche de la sociologie, l'autre de la science politique, en empruntant - plus ou moins - à l'analyse de discours lato sensu), s'intéressent au même objet. On constatera alors que, selon l'angle choisi par l'analyste, les conclusions proposées sont contrastées.
\end{abstract}

Mots clés. - Conflit israélo-palestinien, science politique, sociologie des médias, analyse de discours, réflexivité, champ journalistique, construction et circulation de l'information, objectivité, émotion, internet. 
$\mathrm{D}$ ans cette deuxième série d'« Échanges » consacrée à la critique des médias en temps de conflit, cinq contributeurs poursuivent la réflexion menée dans la livraison précédente par Patrick Charaudeau, Roselyne Koren, Marc Lits, Arnaud Mercier, autour de l'entretien avec Daniel Dayan (2005) par Béatrice Fleury et Jacques Walter. De plusieurs nationalités et/ou issus de champs disciplinaires différents, ils nuancent - comme leurs prédécesseurs - l'argumentation de Daniel Dayan, tout en accentuant la prise en compte des facteurs contextuels dans le traitement médiatique de la seconde Intifada, et en insistant sur la dimension interdisciplinaire d'une analyse de ce type. Une prise en compte d'autant plus prégnante qu'elle a été stimulée par une actualité spécifique, l'entrée de l'armée israélienne au Liban au cours de l'été 2006, un événement concomitant à la rédaction des contributions. Hasard du calendrier que certains ne manquent pas de souligner, parfois même en mettant en relation le traitement médiatique de cet événement avec la seconde Intifada.

Qui sont les présents contributeurs ? Chercheur à l'Institut national de la recherche agronomique et au Centre de sociologie européenne (École des hautes études en sciences sociales), Patrick Champagne a fait ses premières armes à proximité de Pierre Bourdieu, dont il restitue, ici, certains principes théoriques et méthodologiques, utiles en sociologie des médias (voir aussi Champagne, 1990) ; convoquant la théorie des champs, il montre le profit de cette dernière pour étudier le fonctionnement du journalisme. Enseignant l'étude interculturelle des médias à l'université Cardenal Herrera de Valence, Estrella Israel Garzón (200 I) analyse le traitement du conflit israélo-palestinien par les médias espagnols au regard, néanmoins, de paramètres relevant de la globalisation ; au centre de son propos, une notion - l'intra-médiation' définie, dans ce cadre, comme la vision polarisée qui serait à l'œuvre dans les médias et qui consiste à jouer sur le contraste entre victimes (les Palestiniens) et agresseur (Israël) ; au demeurant, plus largement, JeanFrançois Tétu y voit « une première clé de la stratégie, ou de la logique du journalisme contemporain, singulièrement plus facile que de tenter de discuter des responsabilités, des causes et des fins ».Travaillant de longue date sur les agences de presse, Michael Palmer (Boyd, Palmer, 198I) est fin connaisseur de la littérature scientifique anglo-saxonne ${ }^{2}$; un bagage qu'il mobilise de facto afin d'élargir à des considérations internationales le

\footnotetext{
' Inspirée d'H. Borrat (1989 :28-28), cette notion s'applique à la participation des médias au conflit. Elle est à distinguer de celle d'extra-médiation (les médias demeurent en marge et ont une approche se limitant à une narration factuelle) et de celle d'inter-médiation (les médias ambitionnent de jouer un rôle de médiation entre les parties en présence).

${ }^{2}$ C'est cette culture qui le conduit aussi à récuser la distinction, posée par D. Dayan, entre information « journalisée » et information « journalistique ». Pour d'autres raisons, il en va de même de la part de P. Champagne.
} 
propos initial de Daniel Dayan. Même remarque pour la politiste Claire Gabrielle Talon qui, tout en privilégiant un angle différent de celui du chercheur franco-britannique, observe la médiatisation du conflit israélopalestinien au regard des relations entre la France et les pays arabes, voire de la place de la France dans ces pays ; chercheuse au Centre d'études et de recherches internationales (Institut d'études politiques de Paris), elle connaît bien les médias de ces pays, elle qui a réalisé des reportages culturels ou traitant de la mode pour le compte de la chaîne Al-jazira. Quant à Jean-François Tétu, spécialiste de la presse (Mouillaud, Tétu, 1989) et enseignant à l'Institut d'études politiques de Lyon, il décentre le regard porté par Daniel Dayan qui, pour sa part, était axé sur la télévision ; aussi interprète-t-il autrement le traitement journalistique du conflit israélo-palestinien, suggérant que la différence pourrait venir des spécificités des supports concernés.

Si l'on rapproche cette série d'« Échanges » d'autres qui l'ont précédée, on ne peut que constater des lignes de force dans les questionnements des chercheurs, et qui recoupent plusieurs préoccupations exprimées en d'autres occasions. Dans la série traitant de « L'engagement des chercheurs 》 (Questions de communication, 2, 2002 ; Questions de communication, 3, 2003 ; Questions de communication, 4, 2003), c'était le postulat de « neutralité engagée » que convoquait, à l'instar de Max Weber, Nathalie Heinich (2002, 2005), et que discutaient plusieurs chercheurs (voir aussi Meyer, Walter, 2006). On le retrouve ici, bien qu'il ne soit pas explicitement exprimé, en arrière-fond d'une interrogation sur les soubassements de l'analyse de Daniel Dayan : celle-ci relèveraitelle du parti pris ou du travail scientifique ordinaire, avec cependant une dimension critique quant au fonctionnement des médias? Une discussion que mène Patrick Champagne en expliquant l'intérêt à exercer une forme de réflexivité dans l'acte de recherche pour clarifier l'impact de l'engagement et de la distanciation à l'égard de l'objet. Quant à Michael Palmer, il estime que « le scientifique est hybride, tant par sa posture que par sa culture. Face à l'information-news - produit lui aussi hybride, tant par la complexité de sa production, son traitement, sa transmission et sa réception -, le chercheur négocie les prismes de la représentation ». Dans les «Échanges » sur « Les usages du constructivisme »(Questions de communication, 5, 2004 ; Questions de communication, 6, 2004), c'était notamment des aspects concernant l'écriture et le travail journalistiques qui étaient approfondis. Problèmes qui sont ici posés pour déceler, à partir du traitement par les médias du conflit israélo-palestinien, ce qui référerait à une position idéologique ou à des pratiques professionnelles spécifiques. Enfin, dans le développement que Patrick Champagne consacre au rapport « enchanté » au terrain (pour les journalistes et les chercheurs), on retrouve certaines des hypothèses formulées par Stéphane Olivesi (2005) dans les «Échanges » 
consacrés à « Terrain, expérimentation et sciences sociales », quant à l'actuelle fétichisation du terrain, et qui ferait de celui-ci un rite de passage obligé pour les chercheurs, mais non indispensable aux progrès de l'analyse.

Si ces recoupements relèvent de préoccupations sociétales, théoriques et méthodologiques, de façon plus conjoncturelle, ils croisent celles des chercheurs sollicités ici qui se sont tous intéressés au fonctionnement des médias, en situation de crise ou non. Ainsi Patrick Champagne est-il, outre un chercheur attaché à l'analyse des mouvements sociaux, l'un des acteurs d'Acrimed, une association et un site dédiés à l'examen des médias. Quant à Michael Palmer, il compte à son actif des travaux sur le fonctionnement des agences de presse, notamment à l'occasion de la guerre en ex-Yougoslavie (2002, 2003). Pour sa part, Claire Gabrielle Talon connaît - on l'a vu - de l'intérieur les médias arabes. Enfin, JeanFrançois Tétu, avec Isabelle Garcin-Marrou (2003), a travaillé sur le discours de la presse française à l'occasion de la seconde Intifada, et Estrella Israel Garzón (2007) sur le discours de la presse espagnole à propos de l'immigration. Rappelons que nous-mêmes avons mené des recherches sur les médias et la Shoah (Walter, 2005), la guerre d'Algérie (Fleury, 2000), le conflit en ex-Yougoslavie (Pedon, Walter, 2002a ;Pedon, Walter, 2002b), et sur le traitement, par Le Républicain Lorrain, de la bataille de Jénine (Fleury, 2006).

De l'ensemble de ces contributions, nous mettrons en relief les facteurs et théories convoqués pour analyser le passage qui s'opère entre l'événement et sa mise en forme, tant en examinant le lien entre discours politique et contraintes professionnelles qu'en s'attachant aux transformations des préoccupations sociales. Ceci est d'autant plus caractéristique que, dans les pages qui suivent, s'expriment des chercheurs qui, tout en étant de traditions disciplinaires et/ou culturelles différentes (schématiquement, ils se rattachent à deux pôles : l'un proche de la sociologie, l'autre de la science politique, en empruntant - plus ou moins - à l'analyse de discours lato sensu), s'intéressent au même objet. Et nous verrons alors que, selon l'angle choisi par l'analyste, les conclusions proposées sont contrastées.

\section{L'information : entre discours politique et contraintes professionnelles}

Sociologue des médias, nourri aussi de travaux sémiotiques, Daniel Dayan (2005 : 212) qui, pour reprendre la formule de Michael Palmer, s'est jeté dans « la fosse aux lions », explique que « dire qu'un récit fait émerger un événement, c'est en général dire que ce récit suscite la 
curiosité qui rend cet événement notable ou qu'il est aveugle par rapport à d'autres événements. Mais cela peut également vouloir dire qu'un récit puissant est susceptible de provoquer l'existence même de l'événement. La séduction d'un récit peut déjouer les barrières qui se dressent à l'entrée de la sphère publique, faire passer une dramaturgie inconsistante pour un événement valide ». La question n'est plus celle d'une « construction sociale de la réalité », mais celle d'une production de pseudo-événements. Dans ce raisonnement, c'est le rôle des médias qui est pointé, ces derniers fabriquant des événements face auxquels le problème de l'analyste dépasse le fait de savoir s'ils sont ou non en rapport avec la réalité. Ici, on retrouve ce que Daniel J. Boorstin (1963) énonçait pour décrire le pseudo-événement. Prévu et conçu par quelqu'un pour qu'on en parle, celui-ci n'est aucunement spontané et entretient, de ce fait, une relation ambiguë avec la « situation réelle », d'autant qu'il vise « à constituer une prophétie qui se réalise d'ellemême » (ibid. : 33). Selon l'historien américain, ce type de construction correspondrait au « nouveau type d'actualité synthétique qui a envahi notre vie quotidienne. Le préfixe grec "pseudo" signifie "faux", "fait pour tromper" » (ibid. : 30). Si Daniel Dayan (2005 : 216-217) n'évoque pas le « faux » dont l'information serait parée, il traite néanmoins du processus « de filtrage que l'on appelle le gate keeping » et qui « représente une validation de ce qui accède à la sphère publique ». Et c'est notamment sur ce point que plusieurs contributeurs reviennent, chacun s'appuyant sur un point de vue scientifique spécifique, ou bien en mettant en exergue la fonction politique du discours médiatique, ou bien les contraintes professionnelles auxquelles il est soumis.

C'est surtout la dimension politique du discours d'information et de ses conséquences qu'Estrella Israel Garzón met au centre de son propos. La posture de la chercheuse, spécialiste de l'interculturalité, est délibérément critique vis-à-vis des médias espagnols, le traitement des événements ayant trait au Proche-Orient s'inscrivant d'emblée, selon elle, dans une vision conflictuelle avec pour conséquence l'affichage d'un parti pris pour l'un ou l'autre camp. S'appuyant sur les travaux de Mauro Wolf (1987), elle postule l'existence de deux cartes - réelle et médiatique -, la seconde étant conduite à mener une vie qui lui est propre ; par exemple, en multipliant les effets - de mise en scène (dramatisation, répétition), de dénomination des acteurs, d'écriture du récit, de sélection des informations - avec pour conséquence de durcir les différences entre adversaires. En résulte un risque de manipulation dont les journalistes ne sont pas seulement les instigateurs, mais dont ils peuvent eux-mêmes être les « jouets », notamment lorsque l'un des deux camps convoque les médias dans la mise en spectacle de ses propres actions. Elle note également la présence d'un récit simplificateur, à distance d'une réelle compréhension des phénomènes historiques. Aussi, se fondant sur l'analyse d'Adolfo Garcia Ortega (2004), suggère-t- 
elle l'intériorisation, par la société occidentale, d'un schéma mental nourri d'un antisémitisme larvé que masquerait l'accusation de violence dont l'État israélien serait coupable. Une culpabilité qui, à l'aune de la Shoah, verrait des victimes se transformer en bourreaux, et déligitimerait, du même coup, Israël. Une inversion des polarisations que, plus globalement, la chercheuse range sous la notion d'intra-médiation. Toutefois, le renvoi à la période nazie fait l'objet de nuances que pointe Patrick Champagne lorsqu'il relativise la justesse de la référence, par Daniel Dayan, au Journal de Victor Klemperer : du point de vue du sociologue, on ne saurait mettre sur le même plan l'accès à l'espace public dans un régime totalitaire et dans un régime, somme toute, démocratique. Comparaison ne serait pas raison.

En évoquant la « neutralité impossible », cette fois-ci des médias français régulièrement accusés d'être pro-palestiniens, Jean-François Tétu, alliant analyse du discours et science politique, revient lui aussi sur les éléments ayant contribué à un renversement des représentations. Évoquant les propos du général de Gaulle pour qui les Israéliens composeraient un « peuple sûr de lui et dominateur $»^{3}$, le spécialiste de la presse y voit le basculement de l'image de la victime devenue bourreau. Mais s'il éclaire la position des médias par l'histoire politique de la France, il ajoute que cette prise en compte « ne suffit pas à expliquer cette sorte de passion, ni la constance ou l'ampleur de la couverture médiatique ». Et de mentionner le chiffre impressionnant de journalistes à Jérusalem - «900 au printemps 2002, aux pires moments de I'Intifada »-, alors que, dans le même temps, les rédactions désertent d'autres territoires, comme l'Afrique. Pour expliquer ce phénomène, sont invoqués : « Le caractère démocratique d'Israël et la libre circulation des informations ; le fait que ce sol est un berceau de notre civilisation; l'importance, en France, de la communauté juive et des communautés arabo-musulmanes ». Autant de facteurs qui contribuent à rendre difficile la couverture des événements, car ceux-ci sont non seulement très « attendus » par certains lecteurs, mais, de surcroît, sont au centre d'enjeux politiques forts. C'est également ce dont atteste Claire Gabrielle Talon - en politiste ayant croisé les visions médiatiques du conflit en terre arabo-musulmane $e^{4}$ et en France - lorsqu'elle insiste sur la difficulté à laquelle sont confrontés des journalistes devant rendre

\footnotetext{
${ }^{3}$ La formule, prononcée lors d'une conférence de presse (27/1 I/67), est extraite de la phrase suivante : «Certains même redoutaient que les Juifs jusqu'alors dispersés, qui étaient restés ce qu'ils avaient été de tout temps, un peuple d'élite, sûr de lui et dominateur, n'en viennent, une fois qu'ils seraient rassemblés, à changer en ambition ardente et conquérante les souhaits très émouvants qu'ils formaient depuis 19 siècles : "L'an prochain à Jérusalem" ». Cette formule a déclenché de nombreuses réactions indignées tant, pour certains, elle fleurait le maurassisme bon teint...

${ }^{4}$ Le lecteur peu familier des médias dans les mondes arabophones pourra se reporter utilement à G. Kent et M. Palmer (2005).
} 
compte d'une guerre complexe, faite de violences qui s'entremêlent sur le long terme (plus de 60 ans), et qui lui confèrent son caractère unique. Aussi suggère-t-elle qu'aucun fait ne pourra jamais faire l'unanimité, puisque la seule mention de celui-ci « implique l'occultation d'autres faits, le tout relevant d'un débat historique de fond sur les origines du conflit, remontant à la création de l'État d'Israël ». À ce sujet, dans un entretien que Francis Kochert, journaliste au Républicain Lorrain, confiait à deux doctorants du Centre de recherche sur les médiations (université Paul Verlaine-Metz), Hervé Boggio et Mélanie Gérard, celui-ci évoquait le poids que représentait le lectorat issu de la communauté juive implanté de longue date à Metz et l'impact de celui-ci sur le traitement journalistique des événements en lien avec le conflit proche-oriental (Gérard, 2006).

On le voit, si filtre il y a dans le discours journalistique, et s'il est en lien avec des aspects politiques, il ne peut être détaché de paramètres d'ordre professionnel qui, certes, renvoient à la façon dont les journalistes s'organisent, mais aussi à ce qui se joue lorsqu'ils rédigent leurs papiers. Sur ce point, Jean-François Tétu traite de l'émotion, une question qui connaît une percée en sciences sociales (e.g. Livet, 2002 ; Hassoun, 2004, 2005)', et qui a une propension à s'étendre à l'analyse des médias. Un exemple de cet intérêt : l'organisation de plusieurs sessions du $2^{\mathrm{e}}$ Congrès de l'Association française de sociologie - qui s'est tenu à Bordeaux du 5 au 8 septembre 2006, et qui était intitulé « Dire le monde social. Les sociologues face aux discours politiques, économiques et médiatiques »- autour du rapport entre traitement d'un événement et émotion. À noter que Béatrice Fleury (2006) a étudié l'émotion à l'œuvre dans les articles que Francis Kochert avait rédigés lors d'un déplacement en Israël, au cours de la période correspondant à la bataille de Jénine $e^{6}$. Au vu de ces analyses, il apparaît que si l'émotion peut être un procédé inhérent à l'écriture, elle peut aussi surgir lors de la rencontre entre le journaliste et ses sources, et influer sur l'appréciation des faits par ce dernier. Pour Jean-François Tétu, « la situation émouvante tient à la nature de la relation entre le sujet et cette situation, qui provoque peur et colère, joie ou honte, etc. Le support d'information doit évoquer quelque chose qui soit commun au groupe social ». Par ailleurs, sachant que les sentiments se communiquent d'autant mieux que

\footnotetext{
${ }^{5}$ On notera la tenue, à Aix-en-Provence (20-21/10/06), d'un colloque intitulé : « Les mobilisations collectives et les dispositifs de sensibilisation ».

${ }^{6}$ « Dans la nuit du 2 au 3 avril 2002, l'armée israélienne entre dans le camp de Jénine pour y démanteler des organisations palestiniennes. L'accusant d'avoir massacré des centaines de civils, les Palestiniens demanderont qu'une mission de l'ONU soit dépêchée sur place pour constater les faits, projet auquel les Israéliens s'opposeront. Pendant plusieurs mois, l'événement occupera une part non négligeable de l'activité éditoriale française, que ce soit dans la presse nationale ou dans la presse régionale » (Fleury, 2006 : 49).
} 
leurs signes en sont amplifiés, on comprend que les journalistes usent de procédés particuliers, telles la dramatisation et l'esthétisation, la compassion et la dénonciation, ou encore l'appel à la justice...

\section{Quand les préoccupations sociales se transforment}

Mais on ne saurait rendre compte des usages et pratiques des professionnels des médias sans inscrire ceux-ci dans un contexte social particulier - incluant la dimension internationale -, articulé à un cadre médiatique qui dépasse la seule prise en compte de l'événement traité « à chaud ». Par exemple, Claire Gabrielle Talon montre que l'on serait passé, en France, d'un débat focalisé sur l'Intifada à des problèmes touchant plus largement au Proche-Orient, puis à la question de l'antisémitisme. Dans ces conditions, « on comprend combien l'actualité proche-orientale a pu devenir un sujet épineux pour les journalistes ». En effet, « comment présenter l'actualité du conflit israélo-palestinien d'une façon qui, à défaut d'imposer un impossible "consensus", soit satisfaisante pour tous, quand le cours des événements cristallise des oppositions au sein de leur lectorat et divise violemment les téléspectateurs ? ». Aussi, s'intéressant aux événements du Liban de l'été 2006, et comparant la place accordée à celuici à la télévision et dans la presse, démontre-t-elle la part plus restreinte de ce dernier sur les écrans français. En outre, elle constate le traitement grossier dont souffrirait l'actualité proche-orientale, le jugeant « propice à alimenter toutes les méconnaissances ». Un constat qu'elle met en relation avec les ressources moindres des groupes de médias français, comparativement à celles des médias d'autres parties du monde comme le Royaume-Uni, l'Allemagne, les pays arabes. Ceci la conduit à poser un regard pessimiste sur l'information française : « Alors que, partout dans le monde, de grands médias d'information internationale jouent un rôle de plus en plus prépondérant et deviennent des acteurs à part entière des relations internationales, en contribuant à politiser et à mobiliser les opinions publiques à grande échelle et à contrer les politiques, faut-il laisser aux lecteurs du Monde, de Libération du Figaro et du Monde diplomatique, le monopole de l'information sur les grandes crises qui agitent la planète ? ». En s'écartant du point de vue de Daniel Dayan qui s'intéressait surtout à l'information française, elle propose une approche engagée de ce que pourrait être l'information - à distance d'une considération trop étroite de l'objectivité -, et ce au regard du contexte international. Pour elle, en effet, « poser la question de la place de l'actualité proche-orientale sur les écrans français, c'est aussi poser celle du rôle de l'Europe sur la scène procheorientale » (ce qui, du reste, ramène à la problématique de la tripartition des médiations, évoquée par Estrella Israël Garzón). 
Michael Palmer ne dit pas autre chose lorsqu'il écrit : "Pour nous, géopolitique, géo-économie et géo-information sont à penser ensemble. Les visions du monde se croisent, s'interconnectent et connaissent des ratés », non sans relever chez les analystes une certaine « nostalgie des "Grands Récits" » qui, il est vrai, rendait la lecture de la réalité plus simple, au point qu'aujourd'hui, à suivre Jean-François Tétu, on débouche sur « une sorte d'impasse narrative. Sauf à maintenir une grille interprétative très simplifiante (agresseurs vs victimes), il est impossible de construire des rôles actanciels clairs, et les figures semblent sans cesse brouillées ». Mais, pour revenir à l'élargissement de l'analyse vers des considérations internationales, Michael Palmer en ajoute un autre : celui de la diversification des supports pouvant intervenir et interagir sur la matière informationnelle, à savoir la blogosphère, l'en-ligne, la videosphère. Aussi le lecteur peut-il accéder à d'autres sources d'informations, modifiant le rapport qu'il entretient aux médias traditionnels. Mais il peut également s'indigner à plus large échelle et faire pression sur les journalistes : « Reuters et AFP, à Londres, aux ÉtatsUnis, à Paris... relevaient les "bombardements" de leurs sites par des internautes parfois agissant de manière orchestrée : on organise une campagne de cliques (hits) [...] pour critiquer l'écart entre telle photo - de l'angle de tir d'un soldat visant un civil - et la légende qui l'accompagne ; on la scrute au point de déceler des intentions autres que celles que suggère le mot "viser" ».

L'information se révèle donc être une coproduction (Champagne, 1984) où sont associés ceux qui écrivent et ceux qui lisent, mais dans un cadre d'élargissement des frontières, ce qui, notablement, modifie le point de vue de Daniel Dayan. Et si l'on rappelle, comme le fait Patrick Champagne, que le champ journalistique est en lien, mais aussi en compétition, avec les champs économique et politique, on complexifie grandement une approche qui ne verrait dans l'information que le résultat de facteurs idéologiques. Pour le sociologue, « de plus en plus, le journalisme se veut une activité censée dire comment va le monde. Parce que les journalistes se posent comme des témoins honnêtes et sans parti pris et sont, de ce fait, objectivement en concurrence avec les hommes politiques et les spécialistes en sciences sociales, qui, eux aussi, ont vocation à dire le monde social, il importe de rappeler au sociologue des médias que la seule vérité scientifique que l'on peut poser est qu'il existe une lutte pour construire la réalité sociale, pour imposer un point de vue sur la réalité objective, et que cette lutte passe, pour partie, dans le champ journalistique ». Une fois ce préalable posé, Patrick Champagne trace les linéaments d'un monde où s'opposent différents producteurs « pour imposer une représentation du monde ». II le fait en étudiant le traitement, par les médias, des « banlieues en difficulté ». Et si d'aucuns pourraient penser qu'on s'éloigne du sujet, il n'en est rien : le sociologue explique que les représentations n'ayant pas toutes la 
même légitimité, « il n'existe pas de représentation du réel qui soit objective ou acceptée par tous ». Ce qui suppose des tris, sélections, découpages... et un questionnement récurrent du journaliste sur ce qui fera l'information du jour. Or, à ce sujet, le sociologue pose une série de questions, absentes du raisonnement de Daniel Dayan, et qui s'inscrivent dans une option théorique forte, la théorie des champs dont Patrick Champagne aurait aimé que son collègue s'inspire : «Qui est ce journaliste qui s'interroge ainsi, quelle est sa formation, sa position dans le média, la position du média dans le champ journalistique, etc. ? ». Le chercheur de répondre que l'on s'aperçoit « qu'il est là, précisément parce qu'il a les "bonnes" propriétés, celles que demandent un système plus large que l'analyse scientifique doit construire ».

Mais si ces déterminants influent sur le discours, celui-ci n'est pas pour autant figé une fois pour toutes. Par exemple, pour un cas qui intéresse Patrick Champagne - le traitement des incidents dans les banlieues - celuici évolue « notamment au contact des travailleurs sociaux, de leur lecture des sociologues écrivant sur ces problèmes dans les pages "débats" de la presse nationale, etc. ». Et si le sociologue en déduit que « le journaliste n'est qu'un maillon [...] d'un système global » qu'il étend à la société toute entière, on peut ajouter à l'instar de Jean-François Tétu que, selon les thèmes, ce système peut être bien plus large et inclure des événements à grande portée. Ainsi celui du I I-Septembre aurait-il modifié la lecture et l'interprétation d'actes relevant du terrorisme. Si, auparavant, le terrorisme avait pu être envisagé comme une forme de résistance engagée par les faibles, avec le II-Septembre, il perd toute légitimité. Ce qui amène JeanFrançois Tétu à analyser très différemment le traitement de la seconde Intifada dans la presse française que ne le font Daniel Dayan ou Estrella Israel Garzón. Certes, il y voit un cadrage journalistique mettant en exergue l'escalade guerrière d'Israël et qui repose sur « l'opposition entre le "peuple palestinien" et I"'État d'lsraël" ». Mais il y voit aussi « le refus du cadrage "peuple réprimé vs État répressif" ». D'ailleurs, il retrouve ceci en juillet 2006, quand « la figure centrale n'est plus [...] celle de la victime, mais celle de l'échec de ce qui peut se définir comme stratégie (de Yasser Arafat ou d'Ariel Sharon), tactique (des milices, et spécialement du Hamas et du Hezbollah), ou plus généralement, action des Palestiniens. Et cela à cause de la dérive du terrorisme $»$.

\section{Conclusion}

On le constate donc aisément, les analyses sur les médias en temps de conflit, à partir des propos de Daniel Dayan, sont en prise avec une histoire longue (e.g. jusqu'où remonter quand on veut établir les causes d'une action armée ?) et une histoire du temps présent (e.g. comment 
passer du médiatique à l'histoire immédiate ?) qui, l'une et l'autre, nécessitent une vigilance constante de la part des chercheurs : d'une part, l'historiographie évolue, en France comme à l'étranger, ce qui conduit à éviter toute référence exclusive aux seuls travaux hexagonaux; d'autre part, les considérations « à chaud 》 sont minées par les sources auxquelles on est obligé de recourir, soit les médias eux-mêmes. Et quand les pratiques journalistiques font l'objet de l'investigation, ce sont encore d'autres difficultés qui jaillissent. Nombre d'entre elles sont pointées par les contributeurs. Mais il en est d'autres encore qui (re)surgissent à la lecture de leurs articles.

Parmi celles-ci, certaines semblent plus cruciales. Ainsi, tout en faisant des distinctions judicieuses sur les éventuelles variations de traitement, liées aux types de supports (écrits, audiovisuels...), est-on conduit à se demander si les propos tenus sur le journalisme peuvent à ce point être globaux. En effet, le monde journalistique est composé de spécialités et, comme le remarquent fort opportunément les sociologues interactionnistes Rue Bucher et Anselm L. Strauss (196I : 82 et sq.), de segments. Ce vocable désigne des assemblages entre personnes partageant une conception proche de leur activité, ce qui subsume des classifications ordinaires. Par conséquent, de quel journaliste parle-t-on ? Qu'est-ce qui se joue quand on est correspondant permanent, envoyé spécial, journaliste au desk, etc. ? Et peut-on identifier des segments qui orientent la pratique quand il s'agit de couvrir un conflit, en particulier celui qui constitue la matière première de ces «Échanges »? À n'en point douter, des variables influent sur la construction de l'information, de même que, en ce qui concerne les entreprises médiatiques, il y a de réelles probabilités que les conditions d'exercice ne soient pas identiques en fonction de la localisation : par exemple, la presse régionale est soumise à un jeu de contraintes, pour partie différentes, de celui de la presse nationale. Les résultats des premiers travaux sur la couverture de la bataille de Jénine par Le Républicain Lorrain, engagés par le Centre de recherche sur les médiations et le Centre d'étude linguistique des textes et des discours (université Paul Verlaine-Metz), semblent l'attester (voir Fleury, 2006 ; Gérard, 2006).

Ensuite, les prises de position des uns et des autres se font naturellement sur la base d'exemples qui ont été sélectionnés. C'est alors la question du corpus qui est reposée, puisque nous l'avions déjà évoquée dans la précédente livraison de Questions de communication. Certes, dans le cadre de la rubrique «Échanges », on se place habituellement dans une logique de dialogue, d'argumentation et de réfutation d'un texte-source, plus que dans celle de la restitution d'une enquête systématique venant amender les traits saillants de celui-ci. II n'empêche... Chacun délivre son point de vue à partir, certes, d'un modèle théorique et d'un dispositif méthodologique, mais aussi de matériaux empiriques: journal 
personnel, travaux scientifiques antérieurs sur le sujet ou sur un sujet proche (soit factuellement - i.e. un conflit - ; soit structurellement - i.e. le champ journalistique), expérience idiosyncrasique de lecteur de la presse, de téléspectateur ou d'internaute. De fait, le chercheur se fait médiateur entre des discours (au sens large) et des préoccupations scientifiques et sociales : faits langagiers et faits sociaux ne peuvent donc être séparés, y compris dans leur signification ou leur parcours interprétatif. D'où la nécessité d'objectiver les conditions de fabrication dudit corpus, une nécessité de l'ordre de l'évidence lorsqu'on est dans une situation réputée scientifique, qui le serait moins lorsqu'on est en situation d'intervention dans l'espace public. Ce qui n'est pas sans poser problème.

En effet, le point de départ de cette série d'« Échanges » réside bien dans l'énoncé des difficultés à se livrer à une critique publique de pratiques médiatiques, au-delà de la seule sphère académique (tant Daniel Dayan que Pierre Bourdieu les ont rencontrées). II est patent que le principe de l'intervention n'est aucunement récusé et que, plus encore, des solutions sont proposées. II en va ainsi avec l'association Action - Critique - Médias (Acrimed), créée par des proches de Pierre Bourdieu, qui fonctionne comme une sorte d'observatoire : «Née du mouvement social de 1995, dans la foulée de l'Appel à la solidarité avec les grévistes, notre association, pour remplir les fonctions d'un observatoire des médias s'est constituée, depuis sa création en 1996, comme une association-carrefour. Elle réunit des journalistes et salariés des médias, des chercheurs et universitaires, des acteurs du mouvement social et des "usagers" des médias. Elle cherche à mettre en commun savoirs professionnels, savoirs théoriques et savoirs militants au service d'une critique indépendante, radicale et intransigeante » (http://www.acrimed.org [consulté le 3/II/06]). C'est ce qui s'appelle annoncer honnêtement la couleur! Pour autant, cela règle-t-il - en amont - la question du corpus ? Autrement dit, réussir à dépasser le cas particulier par mise en relation sérielle (le corpus étant, du reste, un objet heuristique, plus qu'un moyen), ce qui autorise - sous conditions objectivées - de (prudentes) montées en généralité. À chacun d'en juger. Toutefois, bien souvent, la réaction immédiate et ponctuelle prévaut : elle n'est évidemment pas dénuée d'intérêt ou de pertinence. Elle présente simplement des limites. Et du point de vue du chercheur en sciences sociales, cela conduit à spécifier son mode d'intervention.

Si l'on s'appuie sur les propositions de Nathalie Heinich (2002, 2005) pour clarifier les prises de position dans le débat public, il importe de sérier les différentes postures qui peuvent être adoptées : elles ont un impact sur les discours tenus. Pour ce faire, la sociologue propose des distinctions opératoires. Ainsi, schématiquement, faut-il ne pas confondre une posture de chercheur qui, par un équipement théorico- 
méthodologique adapté, restitue des logiques d'acteurs avec celle de l'expert, qui, pour un tiers, aide à poser un diagnostic et à trouver des solutions à un problème sur lequel il a des compétences; on peut encore se ranger à une posture de « penseur », auquel cas prédomine la formulation de principes et valeurs, argumentés sur la base d'une capacité à manier un savoir. Lorsqu'est en cause un objet du monde ordinaire, tel un débat ou des polémiques sur le traitement médiatique d'un conflit, il s'ensuit que le chercheur est conduit à maintenir son propos dans un cadre énonciatif descriptif ou analytico-descriptif ; il n'est donc ni évaluateur, ni prescripteur, à la différence de l'expert dont c'est la mission ; pour sa part, le penseur a la faculté de tenir un discours normatif et de mettre en circulation des jugements de valeur, sans qu'il soit dans l'obligation de s'appuyer sur des analyses suivant un protocole particulier. Bien entendu, comme toute typologie, celle-ci est instrument de lecture et, à l'épreuve des faits, on peut observer des glissements, si ce n'est des chevauchements entre les postures. En tout cas, c'est, entre autres paramètres, à l'aune de celles-ci que l'on peut comprendre la rationalité de discours émis à partir de cas particuliers, estimés exemplaires.

\section{Références}

Boorstin D. J., 1963, L'image, trad. de l'américain par M.-J. Milcent, Paris, Union générale d'éditions, 1973.

Borrat, H., 1989, El periódico, actor político, Barcelona, G. Pili.

Boyd O., Palmer M., 198I, Le trafic des nouvelles: les agences mondiales d'information, Paris, A. Moreau.

Bucher R., Strauss A. L., 196I, « La dynamique des professions », pp. 67-86, in : Strauss A. L., La trame de la négociation. Sociologie qualitative et interactionnisme, art. trad. de l'américain par J.-M. Chapoulié, éd. par I. Baszanger, Paris, Éd. L'Harmattan, 1992.

Champagne P., 1984, «La manifestation. La production de l'événement politique », Actes de la recherche en sciences sociales, 52-53, pp. I8-41.

— 1990, Faire l'opinion. Le nouveau jeu politique, Paris, Éd. de Minuit.

Dayan D., 2005, « Pour une critique des médias », Questions de communication, 8, pp. 195-222.

Fleury B., 2006, « Le Républicain Lorrain et la bataille de Jénine. De l'événement au témoignage médiatique », Communication et langages, I49, sept., pp. 49-62.

Fleury-Vilatte B., 2000, Mémoire d'Algérie et télévision française : 1962-1992, Paris, Éd. L'Harmattan/INA.

Garcin-Marrou I., Tétu J.-Fr., 2003, « Seconde Intifada et terrorisme. Une analyse des discours de la presse française », Annuaire français des relations internationales, vol. IV, Bruxelles, Bruylant, pp. 849-863. 
Gérard M., 2006, Un envoyé spécial du Républicain Lorrain face au conflit israélopalestinien. Étude des interactions professionnelles dans le cadre de la construction collective de l'information, mémoire de master Médias et médiations, université Paul Verlaine-Metz.

Hassoun J.-P., 2004, « Emotions on the trading floors: social and symbolic expression", pp. 102-120, in : Knorr K., Preda A., eds, The sociology of financial Markets, Oxford University Press.

- 2005, «La place marchande urbaine. Quelques significations sociales », Ethnologie française, I, pp. 5-16.

Heinich N., 2002, « Pour une neutralité engagée », Questions de communication, 2, pp. I 17-127, disponible sur le site ques2com.ciril.fr

- 2005, « Pour en finir avec l'engagement des intellectuels », Questions de communication, 5, pp. 149-160.

Israel Garzón E., 200I, Comunic@ción y Periodismo en una Sociedad global. Comunicar la differencia, México, Trillas.

- 2007, "Les témoignages audiovisuels de l'immigration en Espagne. Un parcours par les médias », in : Fleury B., Walter J., dirs, Médias, migrations, immigrations, Nancy, Presses universitaires de Nancy, à paraître.

Kent G., Palmer J., dirs, 2005, « Mondes arabophones et médias », Questions de communication, 8, pp. 7-1 I2.

Livet P., 2002, Émotions et rationalité morale, Paris, Presses universitaires de France.

Meyer V., Walter J., dirs, 2006, Formes de l'engagement et espace public, Nancy, Presses universitaires de Nancy.

Mouillaud M., Tétu J.-Fr., 1989, Le journal quotidien, Lyon, Presses universitaires de Lyon.

Questions de communication, 2002, 2, «Sur l'engagement des chercheurs 》, pp. 105- I43, disponible sur le site ques2com.ciril.fr

Questions de communication, 2003, 3, « Sur l'engagement des chercheurs (2)», pp. 99-150, disponible sur le site ques2com.ciril.fr

Questions de communication, 2003, 4, « Sur l'engagement des chercheurs (3)», pp. 241-287 disponible sur le site ques2com.ciril.fr

Questions de communication, 2004, 5, «Des usages du constructivisme », pp. $|6|-2||$.

Questions de communication, 2004, 6, « Des usages du constructivisme (2)», pp. $101-177$.

Questions de communications, 2006, 9, « Pour une critique des médias en temps de conflit? », pp. |5|-236.

Olivesi S., 2005, « Le terrain : une mythologie scientifique », Questions de communication, 7, pp. 161-183.

Ortega A. G., 2004, « Antisemitismo », El País, 9 juin, p. 14.

Palmer M., 2002, « L'historien de l'information et les journalistes occidentaux en ex-Yougoslavie », Questions de communication, I, pp. 81-96, disponible sur le site ques2com.ciril.fr 
Pour une critique des médias en temps de conflit? (2)

- 2003, Les mots pour le dire. Correspondants de guerre, journalistes et historiens face aux conflits yougoslaves, Paris, Éd. L'Harmattan.

Pedon É., Walter J., 2002a, dirs, « Les médias et les guerres en ex-Yougoslavie. Débats, théories, méthodes », Questions de communication, I, pp. 3-96, disponible sur le site ques2com.ciril.fr

- 2002b, « Les albums photographiques sur les guerres en ex-Yougoslavie (1990-1994 », Questions de communication, I, pp. 4I-55, disponible sur le site ques2com.ciril.fr

Walter J., 2005, La Shoah à l'épreuve de l'image, Paris, Presses universitaires de France.

Wolf M., 1987, La investigación de masas, Barcelona, Pajdos. 